\title{
A Network-Biology led Computational Drug repurposing Strategy to prioritize therapeutic options for COVID-19
}

Pankaj Khurana ( $\sim$ pkhurana08@gmail.com )

DIPAS

Rajeev Varshney

DIPAS

Aporv gupta

DIPAS

\section{Research Article}

Keywords: Network biology, drug-repurposing

Posted Date: January 12th, 2021

DOl: https://doi.org/10.21203/rs.3.rs-141650/v1

License: (c) (i) This work is licensed under a Creative Commons Attribution 4.0 International License.

Read Full License 


\section{Abstract}

The alarming pandemic situation of novel Severe Acute Respiratory Syndrome Coronavirus 2 (nSARSCoV-2) infection, high drug development cost and slow process of drug discovery have made repositioning of existing drugs for therapeutics a popular alternative. It involves the repurposing of existing safe compounds which results in low overall development costs and shorter development timeline. In the present study, a computational network-biology approach has been used for comparing three candidate drugs i.e. quercetin, N-acetyl cysteine (NAC), and 2-deoxy-glucose (2-DG) to be effectively repurposed against COVID-19. For this, the associations between these drugs and genes of Severe Acute Respiratory Syndrome (SARS) and the Middle East Respiratory Syndrome (MERS) diseases were retrieved and a directed drug-gene-gene-disease interaction network was constructed. Further, to quantify the associations between a target gene and a disease gene, the shortest paths from the target gene to the disease genes were identified. A vector DV was calculated to represent the extent to which a disease gene was influenced by these drugs. Quercetin was quantified as the best among the three drugs, suited for repurposing with DV of -70.19 , followed by NAC with DV of -39.99 and 2-DG with DV of -13.71 . The drugs were also assessed for their safety and efficacy balance (in terms of therapeutic index) using network properties. It was found that quercetin was a forerunner than other two drugs.

\section{Introduction}

The 2019 novel coronavirus (2019-nCoV/SARS-CoV-2), as the cause of coronavirus disease 2019 (abbreviated as COVID-19) has become a health concern to the entire world. As of September 2020 there has been 27 million confirmed cases of COVID-19, including 904,000 deaths for the 2019-nCoV/SARSCoV-2 outbreak worldwide, as reported to WHO. The causative agent is novel Severe acute respiratory syndrome coronavirus 2 (nSARS-CoV-2). Other similar agents were previously known are Middle East Respiratory Syndrome coronavirus (MERS-CoV) and Severe Acute Respiratory Syndrome coronavirus (SARS-CoV). SARS-CoV are enveloped single-stranded, positive-strand ribonucleic acid (RNA) viruses and belong to Coronaviridae family[1]. The structure of SARS-CoV-2 is in close resemblance to that of SARS$\mathrm{CoV}$. The SARS family contains 14 host-binding amino acids. Out of these, 8 amino acids are specifically conserved for SARS-CoV-2 [1]. The binding residues of the family interact with the ACE-2 (Angiotensin converting enzyme-2) of the host directly. The most common symptoms of COVID-19 include fever, malaise, nasal congestion, dry cough, sore throat, dyspnoea, diarrhoea, muscle aches, shortness of breath and multiple organ complications. However, some people serve as asymptomatic carriers of the disease. Currently, no drug or vaccine is available for coping this disease. Precautionary measures are presently the sole approach to prevent person to person transmission.

In the meantime, efforts are underway to develop an effective method of treatment or vaccine. The development of a new molecule is labour-intensive and time-consuming process. It has been assessed that an approved new chemical entity costs more than 1.5 billion and takes 13.5 years from discovery to the market[2]. There are several innovative technologies and approaches to reduce the cost, time and labour and increase the efficiency of drug discovery and development. Drug-repurposing, represented as identification of therapeutically potent molecules against the disease from a library of pre-existing molecule; could considerably shorten the time and reduce the cost compared to de-novo drug discovery and randomized clinical trials. Experimental methodologies for drug repurposing are expensive, laborious and slow[3]. In-silico approaches offer approaches for directed systematic drug repositioning. It offers 
novel testable hypotheses regarding usage, effectiveness, harm and hence supplement evidence generated in randomized controlled trials [3-7].

The repurpose strategy of existing drugs has led to identification of a number of candidate molecules for COVID-19 treatment [8]. As on date, there are nearly 80 clinical trials underway in China on potential treatments [9]. WHO has also recently announced restricted use permission for repurposed anti-HIV, antimalarial, anti-flu and anti-Ebola drugs[10,11] .Three promising molecules that have been proposed as effective therapeutic molecules against COVID-19 are Quercetin (https://clinicaltrials.gov/ct2/show/NCT04377789) N-acetyl Cysteine (https://clinicaltrials.gov/ct2/show/NCT04419025) and 2-Deoxy-D-Glucose (2-DG).

Quercetin and nSARS-Cov-2

Quercetin is a naturally occurring plant pigment (flavonoid) present in colorful fruits and vegetables like berries, onions, red wine, green tea, and apples. It has innumerable health benefits and distinctive biological properties that may improve mental/physical performance and immunity [12]. These properties include anti-carcinogenic, anti-inflammatory, antiviral, antioxidant, psychostimulant, lipid peroxidation inhibition, platelet aggregation and capillary permeability, mitochondrial biogenesis stimulation [13]. It has antioxidant properties and strong ability to fight free-radical molecules. It also has senolytic or anticellular-senescence properties [14]. Thus it is a common ingredient in medicine, supplements, and skincare products. Quercetin and its major metabolites, such as quercetin 3-beta-0-d-glucoside (Q3G, also called isoquercetin), have been found in laboratory studies to inhibit a wide variety of viruses, including SARS-CoV, which is related to COVID-19[15-20]. Some studies have proposed the role of senolytic compounds for the treatment/prevention of corona virus infection [21]. Quercetin is a well-known antisenescene molecule and thus proposed as an effective molecule against COVID-19 [21]. Additionally, docking analysis have ascertained that 'darunavir-quercetin-3- rhamnoside' is a potent and less toxic drug chemical-cocktail/ formulation against SARS-CoV-2-Mpro. Additionally, predicted toxicity and pharmacokinetics profiles also support the hypothesized formulation[22]. In-silico molecular dynamics docking using energy-minimized states of the flavonoids was performed to assess their use against SARS-Cov-2. Quercetin and isoquercetin were among the top 10 flavonoids identified [23]. The $S$ glycoprotein of the virus is cleaved by endosomal proteases cathepsin B/L into S1 and S2 subunits and thereafter the S2 subunit-mediates membrane fusion [24]. Quercetin which is a common and readily available supplement is a strong cathepsin inhibitor with an IC50 in the low micromolar range[25]. Therefore, it is considered as an adjuvant to prevent or to weaken the course of viral infections. The CoV genome encodes two proteases, papain-like protease (PLpro) and 3-chymotrypsin-like protease (3CLpro), also known as the main protease (Mpro), that are important for virus replication[26]. These proteases are among the most promising drug targets. Interestingly, in 3CLpro all residues involved in the dimerization, substrate binding, and catalysis are $100 \%$ conserved between SARS-CoV and SARS-CoV-2[27]. Remarkably, quercetin has a strong binding affinity for 3CLpro[28]. Quercetin thus may block viral replication via inhibition of the viral cysteine protease 3CLpro[29]. Some studies have also proposed quercetin could possibly replace HCQ and be used as prophylactic applications, specifically for the more vulnerable individuals such as the elderly, diabetics, immuno-compromised and those with coronary ailments[29]. A recent study utilises the gene expression profiles of Vitamin D and Quercetin activities to asses them as viable candidates for their potential utility as COVID-19 pandemic mitigation agents [30]. Based on this study, a randomized interventional clinical trial entitled "Phase II Clinical Trial of Estradiol to Reduce Severity of COVID19 Infection in COVID19+ and Presumptive COVID19+ Patients" (https://clinicaltrials.gov/ct2/show/NCT04359329) and two interventional randomized clinical trials evaluating effects of Vitamin D on prevention and treatment of COVID-19 NCT04334005 and NCT04344041 have been listed on ClinicalTrials.gov website. In a recent study, using supercomputerbased in silico drug-docking to the COVID-19 viral spike protein, quercetin was identified among top 5 scoring ligands for viral S-protein-human ACE2 receptor interface[31]. Studies also show that administration of quercetin has resulted in significantly decreased expression of the ACE2 gene during 
differentiation of human intestinal cells [30]. Quercetin may thus inhibit the binding and entry of the virus in the cell $[18,32]$. Molecular docking evaluation of a marine drug metabolite database show that that algae polyphenols and quercetin derivates can be successfully used as treatment against SARS-CoV-2 $[33,34]$. One of the world's most cited scientists, Michel Chrétien, has received 1 million dollars donation to begin a clinical trial of quercetin against COVID-19. Quercetin has already been approved as safe for human consumption by U.S.-based Food and Drug Administration, which means that if the results are encouraging, the treatment will be readily available.

\section{2-DG and nSARS-CoV-2}

2-Deoxy-D-Glucose (2-DG) is a dual decoy of glucose and mannose. It has one of the hydroxyl groups found in the chemical structure of natural glucose replaced by hydrogen. This renders 2-DG ineffective to be converted into energy or support proper glycosylation. It is thus an inhibitor of hexokinase during glycolysis. After the entry of the virus, there is an increased demand of nutrients and energy for the replication of the virus. Thus the host cells have been observed to undergo metabolic reprogramming. 2DG acts as a dual inhibitor of glycolysis as well as glycosylation[35]. This way it hinders in virus replication; its increased energy needs and viral assembly; thus might serve as a probable drug candidate. 2-DG has already been granted permission for clinical trials for other diseases [36-38]. Some in silico studes have tried to investigate glucose anti-metabolite, 2-DG as a repurposed drug for the treatment of novel SARS-CoV-2 virus. These studies have revealed that 2-DG can dock efficiently with viral main protease 3CLpro as well as NSP15 endoribonuclease, and efficiently inactivate these viral receptors leading to incapacitation of the virus[39].A recent study demonstrated that viruses induce an anabolic state in their host cells. This causes these infected cells to upregulate their energy production using glycolysis as compared with their healthy neighbours [35]. Studies show that nontoxic levels 2-DG prevented SARS-CoV-2 replication in Caco-2 cells by blocking carbon metabolism (glycolysis). Importantly, multiple independent studies have shown the disruption of both glycolysis and glycosylation, can have a potent effect against viruses like coronavirus [35, 40-44]. One prodrug of 2-DG, namely, 3,6di-0-acetyl-2-deoxy-d-glucose (WP1122) has been developed in Dr. Waldemar Priebe's laboratory and is owned by the US company Moleculin Biotech. This compound is currently being tested as an antiviral drug for targeting the novel Coronavirus [45] and the company is initiating clinical trials on this drug to fight COVID-19[46]. Proteomics of SARS-CoV-2 infected host cells revealed that the virus reforms central cellular pathways such as translation, splicing, carbon metabolism, protein homeostasis (proteostasis) and nucleic acid metabolism. Inhibitors that target these pathways prevented viral replication in cells. The authors reported that blocking glycolysis with non-toxic concentrations of 2-DG completely prevented SARS-CoV-2 replication in human cells[47]

\section{N-Acetyl Cysteine and nSARS-CoV-2}

$\mathrm{N}$-acetylcysteine (NAC) is reported as a potential candidate for treatment, preventive and/or adjuvant against nSARS-CoV-2 due to a variety of potential properties that include: increases glutathione, improves $T$ cell reposne and anti-inflammation thus reducing the effect of viral induced oxidative stress and cell death[48]. The free sulfhydryl group of NAC helps reduce the disulphide bonds in the mucus glycoproteins matrix and hence helps in reducing mucus viscosity $[49,50]$. Under oxidative stress, NAC also improves redox status by acting as a precursor to cysteine (required for glutathione synthesis), and the ability to replenish thiol pools that help to regulate the redox state [48]. Severe SARS-CoV-2 infection is associated with hallmark responses like high viral load, T cell exhaustion, and high levels of TNF- $\mathbb{Z}$, IL1 $\beta$, IL6, IL10. Thus NAC helps reduce the oxidative stress created by cytokine storm syndrome and production of reactive oxygen species (ROS) may be attenuated by NAC's antioxidant effect[49]. NAC is a precursor of glutathione and has antioxidant properties as it acts as a direct scavenger of Reactive Oxygen Species to regulate redox status and modulate inflammatory response [51]. NAC is also proven to 
decrease airway inflammation and responsiveness in asthma, by modulating the tight junctional protein claudin 18 expression present in airway epithelial cells[52]. The E protein of SARS-CoV interacts with the S-protein through disulphide bonds which can potentially be broken by NAC, thus reducing the viral infectivity[53]. NAC may also decrease pulmonary disease severity by blocking excessive production of angiotensin II[54]. In vitro and clinical studies have shown that NAC can block ACE. This study also demonstrated the decrease of Angiotensin II (ACE2) plasma concentrations just after $2 \mathrm{hrs}$ of NAC administration, suggesting that by blocking $A C E$, it can reduce angiotensin II expression and can inhibit nSARS-CoV-2 viral endocytosis [49]. NAC is known to prevent protein glycosylation. This way it can attenuate the glycosylation events in nSARS-CoV-2 thus decreasing its infectivity. Severe COVID cases exhibit lower GSH and higher ROS levels [55]. NAC is a known inhibitor of NF-kB activation and promotes restauration of thiol pools thus allowing ROS scavenging. This way it may reduce the associated pathologies. NAC has been used in many clinical trials investigating the use of its for treating respiratory illness in humans e.g. Intravenous administered NAC has been used for the treatment of Acute respiratory distress syndrome (ARDS) [48, 56]. Similarly, clinical trial NCT03364218 and NCT03720483 of nebulized NAC has improved arterial oxygen tension in patients suffering from Bronchiolitis; and has attenuated pulmonary fibrosis[57-59]. NAC also has a good safety record in clinical trials. Combination of low oral doses of hydroxychloroquine and intravenous NAC in COVID patient with multisystem organ dysfunction, including acute respiratory insufficiency, yielded positive results. There was a measurable decrease in of several of the inflammatory markers $[60,61]$. Hence a human clinical trial NCT04374461 for using NAC against COVID-19 virus has been recently started sponsored by Prisma Health-Midlands.

Over the years, several approaches are used in computational drug repositioning. Compared to traditional drug repositioning and discovery strategies, network analysis and systems medicine approaches address both depth and breadth of biological knowledge and help to infer molecular connections to discover therapies $[62,63]$. Network based approaches allow widespread, extensive and integrated investigation of multiple biological and molecular datasets to understand vital pathways and can identify functional modules behind multifactorial diseases[64]. These approaches are beneficial to target and potentially help in discovering underlying mechanism responsible for a certain disease pathology and hence may evolve as a potential drug repositioning strategy for complex diseases with varying degree of clinical heterogeneity[65]. Several studies have used different network centric method for drug repurposing e.g. network-based guilt-by-association method (GBA) for drug-disease association prediction-it made suggestions to repurpose drugs based on shared treatment profile from different disease pairs[66]. Another study integrated disease-gene expression signatures, drug targets, disease information, and molecular interaction network to prioritize drug targets for drug repositioning [67].

The present study tries to explore the effectiveness of Quercetin or NAC or 2-DG as a repurposed drug using shortest path in the drug-gene-gene-disease network to identify the extent these drugs affect the SARS/MERS-associated genes. This extent has been quantified and represented in a vector called DV that helps to prioritise these repurposed drugs.

\section{Materials And Methods}

\section{Collection of Disease-genes and construction of Disease-gene network.}

Figure 1 provides a graphical view of the network-based approach to compare Quercetin, NAC, and 2-DG effectiveness against COVID-19 as a repurposed drug. The methodology has been accepted from previous study of Taekeon Lee and Youngmi Yoon et al.[68]. First a directed disease-gene network was constructed. COVID-19 infection is caused by a recently discovered nSARS-CoV-2 that began spreading in December 2019, so there were very few validated-gene-targets available and these were not sufficient to construct a comprehensive disease-gene network. The nSARS-CoV-2 shares an ancestral origin with betacoronavirus like SARS-CoV, MERS-CoV, 229E, HKU1, NL63, and OC43. But SARS-CoV and MERS-CoV 
are the only two betacoronavirus that cause severe respiratory illnesses in humans. Also, nSARS-CoV-2 shares $82 \%$ of its genome with human SARS-CoV and around $43 \%$ identity with MERS-CoV as second best[69]. Therefore SARS and MERS were used to identify the disease associated genes. The disease (SARS and MERS)-gene associations were fetched from DisGeNet[70]. DisGeNet is the largest publicly available repository of genes targets associated with human diseases that have been collected from curated repositories, GWAS catalogs, animal models, and the scientific literature.

The genes associated with these diseases were submitted to Graphite tool to construct a directed disease-gene network[71]. Graphite constructs a directed network by integrating genes and interactions between genes from different pathways repositories such as BioCarta[72], Reactome[73], PID[74], and KEGG[75]. It provides directionality as well as the relationship (positive/negative/neutral) to every interaction between the genes. This relationship was "positive" when activation or expression was recorded, was "negative" when inhibition or repression was recorded, and "neutral" when "binding" was recorded[76]. A directed disease-gene network was thus constructed for further analysis.

\section{Construction of drug-gene-gene-disease directed networks.}

For each drug (Quercetin, NAC, and 2-DG) a comprehensive non-redundant list of associated drug-genes was curated by extensive, manual literature-survey. To increase reliability, the search was constrained to docking and experimentally validated in-vivo and in-vitro studies. These curated drug-gene interactions were assigned as positive/negative/neutral as per drug agonist/antagonist/binding effect on its gene target. These drug-gene targets were mapped to the disease-gene-gene network to construct a directed drug-gene-gene-disease network. Thus three such networks corresponding to three drugs namely quercetin, NAC and 2DG were constructed. The drug-gene-gene-disease directed networks were visualized in Cytoscape.3.6.0.

\section{Shortest paths and DV Calculation from a drug-gene to a disease-gene.}

To calculate the associations between a drug and a disease, the shortest paths from drug-genes to disease-genes were identified. For this, the methodology was adopted from Taekeon Lee \& Youngmi Yoon et al, where they presume that neutral relationships maintain general actions in the body and drugs affect diseases by regulating disease-related genes either by activation or inhibition[68]. Pesca 3.8.0 plugin of Cytoscape was used to identify all the shortest paths between the drug-gene to the disease-gene in the three networks[77].

The extent to which a drug affects the disease-associated genes can be determined using vector DV[68]. To calculate DV, the associations between a drug-gene and a disease-gene in the shortest path were calculated using types and weights of the shortest paths. The type of path $\left(T_{k}\right)$ was calculated by only "positive" and "negative" relationships between genes comprising the path. Thus, 1 and -1 are assigned to each positive and negative edge in the path respectively. The "neutral" path was assigned as 0 to nullify the neutral relationship. The path type $\left(T_{k}\right)$ was defined by multiplying the values corresponding to edges on the path. Path weight $\left(\mathrm{W}_{\mathrm{k}}\right)$, was calculated as the product of the reciprocals of the out-degree of the corresponding node on a path. To consider the extent to which a disease-gene was affected by a drug-gene, $V$ was calculated. $V$ is the sum of all products of weight $(W)$ and path type $(T)$ on the given shortest path. 


$$
V=\sum_{k=1}^{n}\left(\mathrm{~T}_{\mathrm{k}} \times \mathrm{W}_{\mathrm{k}}\right)
$$

To express the effects of quercetin, NAC and 2-DG on the disease, a vector (DV) for each drug-gene-gene-disease path was calculated. DV is denoted as the product of $\left(\mathrm{V}_{\mathrm{k}}\right)$ and the corresponding type of drug-target interaction $\left(\mathrm{Td}_{\mathrm{k}}\right)$.

$$
D V=\sum_{k=1}^{n}\left(\operatorname{sign}\left(\mathrm{Td}_{\mathrm{k}}\right) \mathrm{x} \mathrm{V}_{\mathrm{k}}\right) \quad \operatorname{sign}\left(\mathrm{Td}_{\mathrm{k}}\right)=\left\{\begin{array}{c}
1 \text { if } \mathrm{Td}_{\mathrm{k}}>0 \text { ( positive), } \\
0 \text { if } \mathrm{Td}_{\mathrm{k}}=0 \text { (neutral), } \\
-1 \text { if } \mathrm{Td}_{\mathrm{k}}<0 \text { (negative) }
\end{array}\right.
$$

Network Properties and Biological System Features to assess efficacy and safety balance

The network properties for each drug-gene in the network were generated by PROFEAT [78]. A total of 31 network properties were calculated for subsequent analysis (Table1). These properties are popularly used for analysing complex biological networks. 


\begin{tabular}{|c|c|c|}
\hline 1 & Degree & The number of edges linked to a node \\
\hline 2 & Scaled Connectivity & $\begin{array}{l}\text { The degree of a studied node relative to the most connected node within } \\
\text { the same module }\end{array}$ \\
\hline 3 & Number of Selfloops & The number of edges starting and ending at the same node \\
\hline 4 & Number of Triangles & The number of triangles that include the studied node as a vertex \\
\hline 5 & Z Score & A connectivity index based on degree distribution of a network. \\
\hline 6 & $\begin{array}{l}\text { Clustering } \\
\text { Coefficient }\end{array}$ & The number of the connected pairs between all neighbors of node \\
\hline 7 & $\begin{array}{l}\text { Neighborhood } \\
\text { Connectivity }\end{array}$ & The average connectivity of all neighbors \\
\hline 8 & $\begin{array}{l}\text { Topological } \\
\text { Coefficient }\end{array}$ & $\begin{array}{l}\text { The extent to which a node in network shares interaction partners with } \\
\text { other nodes }\end{array}$ \\
\hline 9 & Interconnectivity & $\begin{array}{l}\text { A connectivity index indicating the quality of the studied nodes being } \\
\text { connected together }\end{array}$ \\
\hline 10 & Bridging Coefficient & $\begin{array}{l}\text { The extent of the studied node lying between any other densely connected } \\
\text { nodes in the network }\end{array}$ \\
\hline 11 & Degree Centrality & The number of links incident upon a studied node \\
\hline 12 & $\begin{array}{l}\text { Avg Shortest Path } \\
\text { Length }\end{array}$ & $\begin{array}{l}\text { The average length of shortest paths between the studied node and all } \\
\text { other ones }\end{array}$ \\
\hline 13 & Distance Sum & The sum of all shortest paths starting from the studied node \\
\hline 14 & Eccentricity & $\begin{array}{l}\text { The maximum non-infinite shortest path length between the studied node } \\
\text { and all other nodes in the network }\end{array}$ \\
\hline 15 & Eccentric & $\begin{array}{l}\text { The absolute difference between nodes' eccentricities } \\
\text { and network's average eccentricity }\end{array}$ \\
\hline 16 & Deviation & The variation between sum of node distances and network unipolarity \\
\hline 17 & Distance Deviation & $\begin{array}{l}\text { The absolute difference between nodes' distance sum and network's } \\
\text { average distance }\end{array}$ \\
\hline 18 & Radiality & $\begin{array}{l}\text { The level of reachability of a studied node via various shortest paths within } \\
\text { the entire network }\end{array}$ \\
\hline 19 & $\begin{array}{l}\text { Closeness Centrality } \\
\text { (avg) }\end{array}$ & $\begin{array}{l}\text { The average number of steps required to reach the studied node from any } \\
\text { node in a network }\end{array}$ \\
\hline 20 & $\begin{array}{l}\text { Closeness Centrality } \\
\text { (sum) }\end{array}$ & $\begin{array}{l}\text { The reciprocal of the sum of the shortest paths between the studied node } \\
\text { and all other nodes in the network }\end{array}$ \\
\hline 21 & $\begin{array}{l}\text { Eccentricity } \\
\text { Centrality }\end{array}$ & The largest geodesic distance between the node and any other node \\
\hline 22 & $\begin{array}{l}\text { Harmonic Closeness } \\
\text { Centrality }\end{array}$ & $\begin{array}{l}\text { The sum of the reciprocals of the average shortest path lengths of each } \\
\text { node in network }\end{array}$ \\
\hline 23 & $\begin{array}{l}\text { Residual Closeness } \\
\text { Centrality }\end{array}$ & The closeness measured by removing the studied node \\
\hline 24 & Load Centrality & The fraction of all the shortest paths that pass through the studied node \\
\hline 25 & $\begin{array}{l}\text { Betweenness } \\
\text { Centrality }\end{array}$ & $\begin{array}{l}\text { The number of times the studied node serving as a linking bridge along } \\
\text { shortest path between any two nodes }\end{array}$ \\
\hline 26 & $\begin{array}{l}\text { Normalized } \\
\text { Betweenness }\end{array}$ & The fraction of network shortest paths that a given protein lies on \\
\hline 27 & Bridging Centrality & The product of the bridging coefficient and betweenness centrality \\
\hline 28 & \begin{tabular}{|l|} 
CurrentFlow \\
Betweenness
\end{tabular} & $\begin{array}{l}\text { A centrality index measuring the level of information travels along all } \\
\text { possible paths within network ( }\end{array}$ \\
\hline 29 & $\begin{array}{l}\text { CurrentFlow } \\
\text { Closeness }\end{array}$ & The variant of current flow betweenness \\
\hline 30 & $\begin{array}{l}\text { Eigenvector } \\
\text { Centrality }\end{array}$ & The sum of its neighbors centrality values \\
\hline
\end{tabular}




\section{Table 1: The 31 network properties calculated for the drug-gene-gene-disease network}

The number of affiliated pathways associated to each drug-gene was obtained from CTD [79]. To asses for collateral effect, the number of human similarity proteins of the gene were determined. This was done by BLAST similarity screening of human proteome (NCBI) with a cutoff (E-value $<0.005)[80]$.

A list of 1,580 human core fitness genes or core-essential-genes was obtained [81]. These are the genes that seem to be constitutively essential for human-system irrespective of context. The top 50 direct interactors of each drug-gene were obtained from STRING[82]. For each drug-gene interactor list, the percentage of core-essential-genes was identified. It is assumed that drugs that target core-essentialgenes of the human proteome might have lower safety levels.

\section{Results And Discussion}

A list of 146 non-redundant disease-genes was identified from the DisGeNet database. The list includes 117 and 53 genes for SARS and MERS respectively with 24 genes common to both the diseases (Supplementary Table S1). A directed disease-gene-gene network having 2394 genes connected via 18810 interactions have constructed by graphite tool. These 18810 interactions of the network were classified into 12306 positive, 3055 negatives, and 3449 neutral interactions.

Further 52, 18, and 40 experimentally validated drug-gene interactions of quercetin, NAC, and 2-DG respectively were manually curated by extensive literature survey (Supplementary Table S2). Also, with each drug its interaction characteristics with the target gene are considered i.e. positive (+1) for agonist, negative (-1) for antagonist and neutral (0) for just binding interaction.

Further, these genes were mapped to the disease-gene-gene network. This way 33, 11, and 31 genes for quercetin, NAC, and 2-DG were retained in the network. Finally, three networks i.e. quercetin-gene-genedisease directed network having 2395 nodes and 18843 interactions(Figure 2), NAC-gene-gene disease directed network having 2395 nodes and 18821 interactions(Figure 3) and 2-DG-gene-gene- disease directed network having 2395 and 18841 interactions (Figure 4) were constructed and visualized in Cytoscape.3.6.0.

\section{Shortest Path and DV vector}

To calculate the association between a drug i.e. quercetin, 2-DG, NAC, and diseases (SARS and MERS), the shortest paths between drug-genes and disease-genes were identified in all the three networks as explained in detail in the methodology section.

In quercetin-gene-gene-disease network, 528 shortest paths were identified (Figure 5). 1\% of the shortest path had common genes interacting with both the drug and the disease directly. These include ESR1, EGFR, ACE, CASP3, BCL2L1 (highlighted as yellow nodes in Figure 5). 7\% of the shortest path had no connecting gene between drug-gene and disease-gene pairs. Lastly, $79 \%$ of these shortest-path contain 1 connecting gene (highlighted as green nodes in Figure 5) between drug-gene and disease-gene, whereas $13 \%$ of these shortest-path contains 2 connecting genes (highlighted as pink nodes in Figure 5) between 
drug-gene and disease-gene (Figure 5). This shows that quercetin could be an effective drug and would be able to affect many disease-associated genes.

Likewise, NAC-gene-gene-disease directed network contains 243 shortest paths of 11 drug-genes (Figure 6). $8 \%$ of these shortest paths have no connecting genes. Whereas, $22 \%$ and $79 \%$ of the shortest path have 1 connecting gene and 2 connecting genes between drug-gene and disease-gene pair respectively.

385 shortest paths of 31 genes targets were identified in the 2-DG-gene-gene-disease directed network (Figure 6). $14 \%$ of the shortest paths in the 2-DG affected network have no connecting genes between drug-gene and disease-gene pair. While $68 \%$ and $18 \%$ of the shortest path contains either 2 connecting genes or 1 connecting gene respectively.

Thus quercetin has a more number of associations with SARS and MERS disease-genes than that of NAC or 2-DG, as shown by the shortest paths between the drug-gene to diasease-gene. In quercetin, $87 \%$ of its total shortest paths have at least 1 connecting gene. While NAC and 2-DG have $30 \%$ and $82 \%$ of their total shortest paths have at least 1 connecting gene respectively. The influence of NAC on disease-genes seem to be complicated as $70 \%$ of the shortest paths have atleast 2 connecting genes between drug-gene and disease-gene.

Further, this effect was quantified and expressed as a vector DV. DV is a vector and denoted the extent to which a disease gene was influenced by a drug. The DV values for quercetin, NAC and 2-DG are -70.19, -39.99 and -13.71 respectively. Quercetin has a greater negative influence on SARS and MERS diseasegene with a DV value of -70.19 (Supplementary Table S3). Whereas NAC and 2-DG show lesser negative influence on SARS and MERS disease-gene target with a DV value of -39.99 (Supplementary Table S4), and -13.71 respectively (Supplementary Table S5).

Thus from the present analysis, quercetin potentially appears to be a better drug that can be repurposed against SARS and MERS than NAC and 2-DG. Thus, quercetin could also be presumably be effectively used against nSARS-CoV- 2 infection because it shares $82 \%$ and $43 \%$ genome similarity with SARS-CoV and MERS-CoV betacoronaviruses.

While analysing the shortest path network of quercetin and 2-DG, some important biological interactions were discovered. Some of these interactions could have therapeutic implications e.g. in quercetin-genegene-disease shortest path network, ESR1, EGFR, ACE, CASP3, BCL2L1 are common direct interactors of both SARS/MERS and quercetin. ESR1 can induce classical IFN-induced antiviral genes, which inhibits the virus entry in the cell $[83,84]$. Quercetin is an agonist of the ESR1 receptor that could help to boost the antiviral response against SARS-CoV or nSARS-CoV-2[85]. EGFR dysregulation protects against pulmonary fibrosis development caused by SARS-CoV and nSARS-CoV-2. Quercetin is an antagonist of EGFR and helps to reduce the risk of developing pulmonary fibrosis during SARS-CoV and nSARS-CoV2[86]. ACE is an important enzyme activated during SARS that converts angiotensin I (AT I) to angiotensin II (AT-II), which further binds to either angiotensin II receptor 1a (AT1aR), leading to tissue damage and lung edema[87]. Quercetin is the inhibitor of the ACE that can help to reduce the tissue damage and formation of lung edema[88]. The protein encoded by ORF-6 present in both SARS-CoV and nSARS-CoV-2 can induce apoptosis through caspase-3 mediated, ER stress, and JNK-dependent pathway[89]. Quercetin inhibits the CASP3 protein and can decrease excessive apoptosis that reduces the formation of ischemic injuries in SARS-CoV patients [90]. It has found that E protein of the SARS-CoV induces the NFKB1 that triggers the expression of pro-inflammatory cytokines such as IL12RB1, IL6, TNF, IL1B, etc[91-93]. This increases the infiltration of more neutrophils in lung tissues. The clinical finding of critical nSARS-CoV-2 patients admitted to intensive care units shows consistent high-level pro-inflammatory cytokines in their plasma[93]. Quercetin has also been found as a direct inhibitor of NFKB1 in the shortest path network that further regulates expression of pro-inflammatory cytokines such as IL12RB1, IL6, TNF, IL1B. and it was observed that treatment with drugs that inhibited NF-KB activation led to a reduction in inflammation and lung pathology in SARS-CoV-infected cultured cells and also increases the mice survival rate[90]. Literature reports have shown that during both SARS-CoV and nSARS-CoV-2 infection there is an

Page 10/27 
overproduction of pro-inflammatory cytokines (TNF, IL-6, and IL-1 $\beta$ ) that results in a cytokine storm [94]. When this high cytokine concentrations get persist over time could lead to an increased risk of vascular hyperpermeability, multiorgan failure, and mortality[94]. Therefore, most of the therapeutical strategies develop until now are directed towards maintaining an adequate inflammatory response for pathogen clearance this includes the use of interleukin-1 inhibitors drug-like anakinra for immune-based therapy of nSARS-CoV-2. Both quercetin and anakinra are antagonists of the human IL-1 receptor[95]. Quercetin and its derivative quercitrin are strong antioxidant agents with anti-inflammatory properties. They are widely used for the treatment of cardiovascular disease, osteoporosis, pulmonary disease, etc[96]. Quercetin reduces the levels of TNF, IL-1 3 , and IL- 6 helps in maintaining the oxidative and inflammatory balance of the body $[96,97]$. Quercetin dysregulating the expression of cytokines pro-inflammatory cytokines like TNF, IL-1 $\beta$, and IL- 6 etc. can be easily visualized in the quercetin targeted shortest path network. Hence both DV vector calculation and biological implication of quercetin targeted shortest path network show quercetin can be effectively used as a therapeutic intervention against nSARS-CoV-2.

$\mathrm{N}$-acetylcysteine (NAC) is a known mucolytic drug for chronic respiratory diseases and has an established safety profile (oral doses of $600 \mathrm{mg} /$ day or $150 \mathrm{mg} / \mathrm{kg}$ in the nebulized format in patients with acute bronchopulmonary diseases like pneumonia, bronchitis, tracheobronchitis). It makes bronchial mucous less viscous and being a cysteine derivative, helps in breaking disulfide bridges between macromolecules, which leads to a reduction in mucus viscosity[98]. NAC is also an inducer of Glutathione synthetase (GSS), which is an important enzyme in glutathione biosynthesis. Glutathione (GSH) in the body has an antioxidant effect and it reduces the formation of proinflammatory cytokines, such as IL-9 and TNF-a, and also has vasodilator properties by increasing cyclic GMP levels and by contributing to the regeneration of endothelial-derived relaxing factor. The inhibition of glutamate receptors (GRIN1, GRIN2A, GRIN2D, GRIN3A) also promotes glutathione synthesis. This interaction can be easily visualized in NAC targeted shortest path network, where NAC promotes the GSS and its downstream signaling and inhibits glutamate receptors (GRIN1, GRIN2A, GRIN2D, GRIN3A). Because of these two properties NAC is already proposed as a potential treatment, preventive, and/or adjuvant against nSARS-CoV-2[49]. This shows that NAC could be an important drug for the treatment of nSARS-CoV-2 infection but DV calculation shows it presumably would be less effective as an nSARS-CoV-2 therapeutic agent as compared to quercetin.

2-DG is a mimicking agent of glucose and is an antagonist of glucokinase/hexokinase (GCK/HCK), an enzyme that converts glucose to glucose- 6 phosphate in the glycolysis cycle [99]. It is also a rate-limiting step of the oxidative metabolism. The inhibition of GCK reduces the ATP levels in the cells. Hence there would be more AMP levels than ATP levels in the cells. This leads to the activation of AMP-Activated protein kinases (PRKAG, PRKAB, PRKAA) [100]. These AMP-activated kinases inhibit Janus kinase (JAK1) and activate STAT transcription factors, especially Signal transducer and activators of transcription-3 (STAT3) [101]. STAT3 regulates cell growth, differentiation, and apoptosis[102]. During SARS infection in Vero cells, it was found that the induction of STAT3 leads to cell apoptosis, thus helping to stop viral replication [103]. This interaction can be easily visualized in 2-DG targeted shortest path network where 2DG inhibits the GCK and its downstream signaling and leads to the activation of STAT3 through different AMP-activated kinase intermediates. Though 2-DG has a therapeutic effect against SARS, it follows a more complex route to target genes associated with SARS and MERS. Hence as hinted by DV calculation, presumably it might not be as effective as quercetin and NAC as a re-purposed drug for COVID-19.

\section{Network properties and Biological System Features to assess safety and efficacy balance}

Therapeutic Index is a measure of relative safety of a drug. It is calculated as ratio of the dose that produces toxicity to the dose needed to produce the desired therapeutic response. Drugs with $\mathrm{Tl} \leq 3$ are considered less safe and referred to as Narrow Therapeutic Index (NTI) drugs. Drugs with $\mathrm{TI}>3$ are considered better than NTI on safety standards and referred to as Not -Narrow Therautic Index (NNTI) drugs. However, determination of $\mathrm{TI}$ is very complicated for many drugs and is also highly susceptible to the variations of drug responses.

Page $11 / 27$ 
The efficacy-safety balance of a drug may be inferred from the network properties and biological system profile of the drug-genes[104]. So, an effort was made to assess the safety efficacy balance (i.e. NTI or $\mathrm{NNTI}$ ) of the three drugs (quercetin, NAC and 2-DG) in the drug-gene-gene-disease network. Several connectivity and adjacency-based network properties, properties based on shortest path length, have been found to be significantly different ( $p$-value $<0.05$ ) between the targets of NTI and NNTI drugs[104] (Table 2). Network properties were grouped based on their innate mutual dependence are highlighted in similar colours. 


\begin{tabular}{|c|c|c|c|c|c|c|}
\hline & Parameter & NNTI & NTI & Quercetin & NAC & 2DG \\
\hline 1 & Closeness Centrality (avg) & Smaller & Larger & 0.241 & 0.264 & 0.272 \\
\hline 2 & Harmonic Closeness Centrality & Smaller & Larger & 635.697 & 695.509 & 715.510 \\
\hline 3 & Residual Closeness Centrality & Smaller & Larger & 180.569 & 223.146 & 237.773 \\
\hline 4 & Avg Shortest Path Length & Larger & Smaller & 4.242 & 3.846 & 3.709 \\
\hline 5 & Deviation & Larger & Smaller & 3493.062 & 2473.818 & 2156.581 \\
\hline 6 & Distance Deviation & Larger & Smaller & 1247.556 & 1572.156 & 1789.97 \\
\hline 7 & Distance Sum & Larger & Smaller & 13093.437 & 9241.818 & 8910.581 \\
\hline 8 & Betweenness Centrality & Smaller & Larger & 0.002 & 0.0069 & 0.0065 \\
\hline 9 & CurrentFlow Betweenness & Smaller & Larger & 0.0049 & 0.01 & 0.0099 \\
\hline 10 & Load Centrality & Smaller & Larger & 0.0020 & 0.0070 & 0.0068 \\
\hline 11 & Normalized Betweenness & Smaller & Larger & 0.0121 & 0.03636 & 0.03280 \\
\hline 12 & Eccentric & Smaller & Larger & 0.574 & 0.433 & 0.593 \\
\hline 13 & Eccentricity & Larger & Smaller & 9.0625 & 9.1818 & 8.9354 \\
\hline 14 & Eccentricity Centrality & Smaller & Larger & 0.1111 & 0.1091 & 0.1123 \\
\hline 15 & Degree & Smaller & Larger & 21.5312 & 32.1818 & 25.9032 \\
\hline 16 & Degree centrality & Smaller & Larger & 0.0088 & 0.0132 & 0.0107 \\
\hline 17 & Z score & Smaller & Larger & 0.0180 & 0.04490 & 0.0287 \\
\hline 18 & \begin{tabular}{|l} 
Radiality \\
\end{tabular} & Smaller & Larger & 0.7505 & 0.7810 & 0.7916 \\
\hline 19 & \begin{tabular}{|l} 
Clustering coefficient \\
\end{tabular} & Larger & Smaller & 0.2505 & 0.432 & 0.2311 \\
\hline 20 & Interconnectivity & Larger & Smaller & 0.3883 & 0.4976 & 0.3312 \\
\hline 21 & Neighbor connectivity & Smaller & Larger & 35.0512 & 44.2683 & 37.0124 \\
\hline 22 & Topological coefficient & Larger & Smaller & 0.3444 & 0.2276 & 0.2091 \\
\hline \multicolumn{7}{|c|}{ Biological System Features } \\
\hline 1 & $\begin{array}{l}\text { No. of pathways affiliated by the primary } \\
\text { therapeutic target }\end{array}$ & Smaller & Larger & 45.59 & 54 & 59.51 \\
\hline 2 & $\begin{array}{l}\text { No. of similarity proteins outside the } \\
\text { target family }\end{array}$ & Smaller & Larger & 32.53 & 53.27 & 38.45 \\
\hline 3 & $\begin{array}{l}\text { Number of director interactors which are } \\
\text { core essential genes }\end{array}$ & Smaller & Larger & 7.6 & 24.02 & 9.1 \\
\hline
\end{tabular}

Table 2: The network properties that have been found to be significantly different ( $p$-value $<0.05$ ) between the targets of NTI and NNTI drugs. Network properties were grouped based on their innate mutual dependence at highlighted in similar colors. The values larger/smaller in NTI/NNTI drugs is listed. The corresponding average values of the target genes of quercetin, NAC and 2-DG are also tabulated. The profiles which match the NNTI are highlighted in red color. 
Some key features such as average shortest path length show increase from the targets of NTI drug to that of NNTI one[104]. Among the three, quercetin showed the highest average shortest path length, followed by NAC and 2-DG (Table 2). Also some others such as average closeness centrality demonstrated a decrease from the targets of NTI drug to that of NNTI drugs[104]. The lower value of average closeness centrality of drug-target has been shown to demonstrate a less lethality risk [105]. The interconnectivity values were lower for lethal diseases like cardiovascular and oncogenic diseases [106]. Quercetin had the smallest average closeness centrality among the three (Table 2).

Based on these parameters, Quercetin and NAC were found to be closer to the profile of NNTI drugs. Properties which determine the connectivity of the target (i.e average shortest path length, bridgingcoefficient, interconnectivity) showed an increasing trend from the targets of NTI to NNTI drugs[104]. These parameters for quercetin, NAC and 2-DG are depicted in Figure 8. Average shortest path length, bridging-coefficient were highest in quercetin; whereas interconnectivity was highest in NAC.

Parameters which determine the centrality of the target in the network (i.e. average closeness centrality, degree, radiality) showed a decreasing trend from the targets of NTI to NNTI drugs[104]. These parameters for quercetin, NAC and 2-DG are depicted in Figure 8. Average closeness centrality, degree, radiality were lowest in quercetin.

Biological system properties (i.e. affiliated pathways, number of similarity proteins) also showed decreasing trend from the targets of NTI to NNTI drugs[104]. The affiliated pathways and similarity proteins were lowest in quercetin targets (Table 2).

It has been proven that the TI-related mechanism could be a result of synergistically effects among these eight features (Figure)[104]. As per these parameters, among the three, quercetin followed the trends of NNTI drug. Other studies have also shown that number of similarity proteins and affiliated pathways as a good indicator of target drugability $[107,108]$.

Thus the targets of NTI drugs were highly centralized and connected in network, and the numbers of similarity proteins and target-affiliated pathways were higher than those of NNTI drugs [104].

A list of 1580 core-essential-genes was obtained[109]. These genes without any context-dependence have been important for survival. For each drug-gene, its top 50 direct interactors were obtained from STRING. The percentage of these direct interactors which were part of core-essential-genes were identified. It is presumed that the drugs which target the core-essential-genes would be NTI drugs. It was found that quercetin had the minimum number of core-essential-gene interactors (Table 2).

\section{Conclusions}

In this study, we compare three drug candidates i.e. quercetin, NAC, and 2-DG to be repositioned for nSARS-CoV-2 by exploring associations between drugs and disease genes derived from a directed druggene-gene-disease network using the shortest path. Further, we have quantified the extent of a diseasegene influenced by a drug in the directed drug-gene-gene-disease network through a vector called DV. DV calculation showed that quercetin has a greater therapeutic influence on the SARS and MERS associated genes, followed by NAC and 2-DG. The shortest path calculation shows that both NAC and 2-DG follow a complex route to effect the SARS and MERS genes as compared to quercetin. Also, the shortest path calculation of quercetin revealed five genes ESR1, EGFR, ACE, CASP3, BCL2L1 that are also directly associated with SARS and MERS. As nSARS-CoV-2 shares an ancestral origin and genome with 
betacoronavirus like SARS-CoV, MERS-CoV, therefore, quercetin could potentially be a more potent drug candidate that could be effective against nSARS-CoV-2 infection. Further the efficacy-safety balance of these drugs were assessed by connectivity and centrality of the drug-targets in the drug-gene-disease network. Quercetin was found to follow the pattern of a better and safer drug.

\section{References}

1. Pal, M., et al., Severe Acute Respiratory Syndrome Coronavirus-2 (SARS-CoV-2): An Update. Cureus, 2020. 12(3): p. e7423.

2. Paul, S.M., et al., How to improve R\&D productivity: the pharmaceutical industry's grand challenge. Nat Rev Drug Discov, 2010. 9(3): p. 203-14.

3. Cheng, F., In Silico Oncology Drug Repositioning and Polypharmacology. Methods Mol Biol, 2019. 1878: p. 243-261.

4. Cheng, F., et al., Individualized network-based drug repositioning infrastructure for precision oncology in the panomics era. Brief Bioinform, 2017. 18(4): p. 682-697.

5. Cheng, F., J.L. Murray, and D.H. Rubin, Drug Repurposing: New Treatments for Zika Virus Infection? Trends Mol Med, 2016. 22(11): p. 919-921.

6. Santos, R., et al., A comprehensive map of molecular drug targets. Nat Rev Drug Discov, 2017. 16(1): p. 19-34.

7. Cheng, F., et al., Network-based approach to prediction and population-based validation of in silico drug repurposing. Nat Commun, 2018. 9(1): p. 2691.

8. Ciliberto, G., R. Mancini, and M.G. Paggi, Drug repurposing against COVID-19: focus on anticancer agents. J Exp Clin Cancer Res, 2020. 39(1): p. 86.

9. Maxmen, A., More than 80 clinical trials launch to test coronavirus treatments. Nature, 2020. 578(7795): p. 347-348.

10. Senathilake, K.S., S.; Tennekoon, Virtual Screening of Inhibitors Against Spike Glycoprotein of SARSCoV-2: A Drug Repurposing Approach. Preprints 2020, 2020.

11. Guo, Y.R., et al., The origin, transmission and clinical therapies on coronavirus disease 2019 (COVID19) outbreak - an update on the status. Mil Med Res, 2020. 7(1): p. 11.

12. Li, Y., et al., Quercetin, Inflammation and Immunity. Nutrients, 2016. 8(3): p. 167.

13. Chen, S., et al., Therapeutic Effects of Quercetin on Inflammation, Obesity, and Type 2 Diabetes. Mediators Inflamm, 2016. 2016: p. 9340637.

14. Malavolta, M., et al., Pleiotropic Effects of Tocotrienols and Quercetin on Cellular Senescence: Introducing the Perspective of Senolytic Effects of Phytochemicals. Curr Drug Targets, 2016. 17(4): p. 447-59.

15. Qiu, X., et al., Prophylactic Efficacy of Quercetin 3-beta-O-d-Glucoside against Ebola Virus Infection. Antimicrob Agents Chemother, 2016. 60(9): p. 5182-8. 
16. Colunga Biancatelli, R.M.L., et al., Quercetin and Vitamin C: An Experimental, Synergistic Therapy for the Prevention and Treatment of SARS-CoV-2 Related Disease (COVID-19). Front Immunol, 2020. 11: p. 1451.

17. Polansky, H. and G. Lori, Coronavirus disease 2019 (COVID-19): first indication of efficacy of GeneEden-VIR/Novirin in SARS-CoV-2 infection. Int J Antimicrob Agents, 2020. 55(6): p. 105971.

18. Nguyen, T.T., et al., Flavonoid-mediated inhibition of SARS coronavirus 3C-like protease expressed in Pichia pastoris. Biotechnol Lett, 2012. 34(5): p. 831-8.

19. Mani, J.S., et al., Natural product-derived phytochemicals as potential agents against coronaviruses: A review. Virus Res, 2020. 284: p. 197989.

20. Luo, E., et al., Treatment efficacy analysis of traditional Chinese medicine for novel coronavirus pneumonia (COVID-19): an empirical study from Wuhan, Hubei Province, China. Chin Med, 2020.15 . p. 34.

21. Sargiacomo, C., F. Sotgia, and M.P. Lisanti, COVID-19 and chronological aging: senolytics and other anti-aging drugs for the treatment or prevention of corona virus infection? Aging (Albany NY), 2020. 12(8): p. 6511-6517.

22. Shasank S. Swain, S.R.S., Alaka Sahoo, Tahziba Hussain, Sanghamitra Pati Molecular dockingsimulation edge assessment of potential and less-toxic 'anti- HIV-drug and phyto-flavonoid' combination against COVID-19. Preprints, 2020.

23. Peterson, L., COVID-19 and Flavonoids: In Silico Molecular Dynamics Docking to the Active Catalytic Site of SARS-CoV and SARS-CoV-2 Main Protease. 2020.

24. Turk, V., et al., Cysteine cathepsins: from structure, function and regulation to new frontiers. Biochim Biophys Acta, 2012. 1824(1): p. 68-88.

25. Wang, L., et al., Quercetin, a flavonoid with anti-inflammatory activity, suppresses the development of abdominal aortic aneurysms in mice. Eur J Pharmacol, 2012. 690(1-3): p. 133-41.

26. Baez-Santos, Y.M., S.E. St John, and A.D. Mesecar, The SARS-coronavirus papain-like protease: structure, function and inhibition by designed antiviral compounds. Antiviral Res, 2015. 115: p. 21-38.

27. Chen, Y.W., C.B. Yiu, and K.Y. Wong, Prediction of the SARS-CoV-2 (2019-nCoV) 3C-like protease (3CL (pro)) structure: virtual screening reveals velpatasvir, ledipasvir, and other drug repurposing candidates. F1000Res, 2020. 9: p. 129.

28. Huynh, T., H. Wang, and B. Luan, In Silico Exploration of the Molecular Mechanism of Clinically Oriented Drugs for Possibly Inhibiting SARS-CoV-2's Main Protease. J Phys Chem Lett, 2020. 11(11): p. 4413-4420.

29. Depfenhart, M., et al., Potential new treatment strategies for COVID-19: is there a role for bromhexine as add-on therapy? Intern Emerg Med, 2020. 15(5): p. 801-812.

30. Glinsky, G.V., Tripartite Combination of Candidate Pandemic Mitigation Agents: Vitamin D, Quercetin, and Estradiol Manifest Properties of Medicinal Agents for Targeted Mitigation of the COVID-19 Pandemic Defined by Genomics-Guided Tracing of SARS-CoV-2 Targets in Human Cells. Biomedicines, 2020. 8(5). 
31. Smith M, S.J., Repurposing Therapeutics for COVID-19: Supercomputer-Based Docking to the SARSCoV-2 Viral Spike Protein and Viral Spike Protein-Human ACE2 Interface. ChemRxiv, 2020.

32. Yi, L., et al., Small molecules blocking the entry of severe acute respiratory syndrome coronavirus into host cells. J Virol, 2004. 78(20): p. 11334-9.

33. Antonio, A.d.S., L.S.M. Wiedemann, and V.F. Veiga-Junior, Natural products' role against COVID-19. RSC Advances, 2020. 10(39): p. 23379-23393.

34. Gentile, D., et al., Putative Inhibitors of SARS-CoV-2 Main Protease from A Library of Marine Natural Products: A Virtual Screening and Molecular Modeling Study. Mar Drugs, 2020. 18(4).

35. Gualdoni, G.A., et al., Rhinovirus induces an anabolic reprogramming in host cell metabolism essential for viral replication. Proc Natl Acad Sci U S A, 2018. 115(30): p. E7158-E7165.

36. Mohanti, B.K., et al., Improving cancer radiotherapy with 2-deoxy-D-glucose: phase I/II clinical trials on human cerebral gliomas. Int J Radiat Oncol Biol Phys, 1996. 35(1): p. 103-11.

37. Vijayaraghavan, R., et al., Acute toxicity and cardio-respiratory effects of 2-deoxy-D-glucose: a promising radio sensitiser. Biomed Environ Sci, 2006. 19(2): p. 96-103.

38. Dwarakanath, B.S., et al., Clinical studies for improving radiotherapy with 2-deoxy-D-glucose: present status and future prospects. J Cancer Res Ther, 2009. 5 Suppl 1: p. S21-6.

39. Balkrishna, A.T., Pallavi \& Singh, Shivam \& Dev, Swami Narsingh \& Jain, Viney \& Varshney, Anurag \& Sharma, Rakesh, Glucose antimetabolite 2-Deoxy-D-Glucose and its derivative as promising candidates for tackling COVID-19: Insights derived from in silico docking and molecular simulations. 2020.

40. Fontaine, K.A., et al., Dengue virus induces and requires glycolysis for optimal replication. J Virol, 2015. 89(4): p. 2358-66.

41. Wang, Y., et al., Triggering unfolded protein response by 2-Deoxy-D-glucose inhibits porcine epidemic diarrhea virus propagation. Antiviral Res, 2014. 106: p. 33-41.

42. Schmidt, M.F., R.T. Schwarz, and C. Scholtissek, Interference of nucleoside diphosphate derivatives of 2-deoxy-D-glucose with the glycosylation of virus-specific glycoproteins in vivo. Eur J Biochem, 1976. 70(1): p. 55-62.

43. Maehama, T., et al., Selective down-regulation of human papillomavirus transcription by 2deoxyglucose. Int J Cancer, 1998. 76(5): p. 639-46.

44. Leung, H.J., et al., Activation of the unfolded protein response by 2-deoxy-D-glucose inhibits Kaposi's sarcoma-associated herpesvirus replication and gene expression. Antimicrob Agents Chemother, 2012. 56(11): p. 5794-803.

45. Pajak, B., et al., 2-Deoxy-d-Glucose and Its Analogs: From Diagnostic to Therapeutic Agents. Int J Mol Sci, 2019. 21(1).

46. Priebe, W., Zielinski, R., Fokt, I., Felix, E., Radjendirane, V., Arumugam, J., Tai Khuong, M., Krasinski, M., Skora, S. (2018). EXTH-07. Design and evaluation of WP1122, in inhibitor of glycolysis with 
increased CNS uptake, Design and evaluation of WP1122, in inhibitor of glycolysis with increased CNS uptake. Neuro-Oncology, 2018. 20(6).

47. Bojkova, D., et al., Proteomics of SARS-CoV-2-infected host cells reveals therapy targets. Nature, 2020. 583(7816): p. 469-472.

48. Poe, F.L. and J. Corn, N-Acetylcysteine: A potential therapeutic agent for SARS-CoV-2. Med Hypotheses, 2020. 143: p. 109862.

49. Jorge-Aaron, R.M. and M.P. Rosa-Ester, N-acetylcysteine as a potential treatment for COVID-19. Future Microbiol, 2020. 15: p. 959-962.

50. Aldini, G., et al., N-Acetylcysteine as an antioxidant and disulphide breaking agent: the reasons why. Free Radic Res, 2018. 52(7): p. 751-762.

51. Andreou, A., et al., COVID-19: The Potential Role of Copper and N-acetylcysteine (NAC) in a Combination of Candidate Antiviral Treatments Against SARS-CoV-2. In Vivo, 2020. 34(3 Suppl): p. 1567-1588.

52. Lee, P.H., J. Hong, and A.S. Jang, $N$-acetylcysteine decreases airway inflammation and responsiveness in asthma by modulating claudin 18 expression. Korean J Intern Med, 2020. 35(5): p. 1229-1237.

53. Schoeman, D. and B.C. Fielding, Coronavirus envelope protein: current knowledge. Virol J, 2019. 16(1): p. 69.

54. Ullian, M.E., et al., N-acetylcysteine decreases angiotensin II receptor binding in vascular smooth muscle cells. J Am Soc Nephrol, 2005. 16(8): p. 2346-53.

55. Polonikov, A., Endogenous Deficiency of Glutathione as the Most Likely Cause of Serious Manifestations and Death in COVID-19 Patients. ACS Infect Dis, 2020. 6(7): p. 1558-1562.

56. Miller, A.C., et al., Influence of nebulized unfractionated heparin and $\mathrm{N}$-acetylcysteine in acute lung injury after smoke inhalation injury. J Burn Care Res, 2009. 30(2): p. 249-56.

57. Ueno, O., L.N. Lee, and P.D. Wagner, Effect of $\mathrm{N}$-acetylcysteine on gas exchange after methacholine challenge and isoprenaline inhalation in the dog. Eur Respir J, 1989. 2(3): p. 238-46.

58. Masoompour, S.M., A. Anushiravani, and A. Tafaroj Norouz, Evaluation of the Effect of Nebulized NAcetylcysteine on Respiratory Secretions in Mechanically Ventilated Patients: Randomized Clinical Trial. Iran J Med Sci, 2015. 40(4): p. 309-15.

59. Hagiwara, S.I., Y. Ishii, and S. Kitamura, Aerosolized administration of N-acetylcysteine attenuates lung fibrosis induced by bleomycin in mice. Am J Respir Crit Care Med, 2000. 162(1): p. 225-31.

60. Ibrahim, H., et al., Therapeutic blockade of inflammation in severe COVID-19 infection with intravenous N-acetylcysteine. Clin Immunol, 2020. 219: p. 108544.

61. Puyo C, K.D., Saddi V Case Report: Use of hydroxychloroquine and N-acetylcysteine for treatment of a COVID-19 patient. F1000Research, 2020. 9(491).

62. Shameer, K., et al., A Network-Biology Informed Computational Drug Repositioning Strategy to Target Disease Risk Trajectories and Comorbidities of Peripheral Artery Disease. AMIA Jt Summits Transl 
Sci Proc, 2018. 2017: p. 108-117.

63. Li, J., et al., A survey of current trends in computational drug repositioning. Brief Bioinform, 2016. 17(1): p. 2-12.

64. Jeong, H., et al., Lethality and centrality in protein networks. Nature, 2001. 411(6833): p. 41-2.

65. Schadt, E.E., S.H. Friend, and D.A. Shaywitz, A network view of disease and compound screening. Nat Rev Drug Discov, 2009. 8(4): p. 286-95.

66. Chiang, A.P. and A.J. Butte, Systematic evaluation of drug-disease relationships to identify leads for novel drug uses. Clin Pharmacol Ther, 2009. 86(5): p. 507-10.

67. Zhang, T.T., et al., Network-based drug repositioning: A novel strategy for discovering potential antidepressants and their mode of action. Eur Neuropsychopharmacol, 2018. 28(10): p. 1137-1150.

68. Lee, T. and Y. Yoon, Drug repositioning using drug-disease vectors based on an integrated network. BMC Bioinformatics, 2018. 19(1): p. 446.

69. Xu, J., et al., Systematic Comparison of Two Animal-to-Human Transmitted Human Coronaviruses: SARS-CoV-2 and SARS-CoV. Viruses, 2020. 12(2).

70. Pinero, J., et al., DisGeNET: a comprehensive platform integrating information on human diseaseassociated genes and variants. Nucleic Acids Res, 2017. 45(D1): p. D833-D839.

71. Sales, G., et al., graphite - a Bioconductor package to convert pathway topology to gene network. BMC Bioinformatics, 2012. 13: p. 20.

72. Nishimura, D., BioCarta. Biotech Software \& Internet Report, 2004. 2(3): p. 117-120.

73. Fabregat, A., et al., Reactome graph database: Efficient access to complex pathway data. PLoS Comput Biol, 2018. 14(1): p. e1005968.

74. Schaefer, C.F., et al., PID: the Pathway Interaction Database. Nucleic Acids Res, 2009. 37(Database issue): p. D674-9.

75. Kanehisa, M., et al., KEGG: new perspectives on genomes, pathways, diseases and drugs. Nucleic Acids Res, 2017. 45(D1): p. D353-D361.

76. Yu, H., et al., Prediction of drugs having opposite effects on disease genes in a directed network. BMC Syst Biol, 2016. 10 Suppl 1: p. 2.

77. Scardoni, G., et al., Finding the shortest path with PesCa: a tool for network reconstruction. F1000Res, 2015. 4: p. 484.

78. Zhang, P., et al., A protein network descriptor server and its use in studying protein, disease, metabolic and drug targeted networks. Brief Bioinform, 2017. 18(6): p. 1057-1070.

79. Davis, A.P., et al., The Comparative Toxicogenomics Database: update 2019. Nucleic Acids Res, 2019. 47(D1): p. D948-D954.

80. Altschul, S.F., et al., Basic local alignment search tool. J Mol Biol, 1990. 215(3): p. 403-10.

81. Hart, T., et al., High-Resolution CRISPR Screens Reveal Fitness Genes and Genotype-Specific Cancer Liabilities. Cell, 2015. 163(6): p. 1515-26. 
82. von Mering, C., et al., STRING: a database of predicted functional associations between proteins. Nucleic Acids Res, 2003. 31(1): p. 258-61.

83. Zhou, Y., et al., Network-based drug repurposing for novel coronavirus 2019-nCoV/SARS-CoV-2. Cell Discov, 2020. 6: p. 14.

84. Kovats, S., Estrogen receptors regulate innate immune cells and signaling pathways. Cell Immunol, 2015. 294(2): p. 63-9.

85. van der Woude, H., et al., The stimulation of cell proliferation by quercetin is mediated by the estrogen receptor. Mol Nutr Food Res, 2005. 49(8): p. 763-71.

86. Baby, B., P. Antony, and R. Vijayan, Interactions of quercetin with receptor tyrosine kinases associated with human lung carcinoma. Nat Prod Res, 2018. 32(24): p. 2928-2931.

87. Nicholls, J. and M. Peiris, Good ACE, bad ACE do battle in lung injury, SARS. Nat Med, 2005. 11(8): p. 821-2.

88. Muhammad, S.A. and N. Fatima, In silico analysis and molecular docking studies of potential angiotensin-converting enzyme inhibitor using quercetin glycosides. Pharmacogn Mag, 2015. 11(Suppl 1): p. S123-6.

89. Ye, Z., et al., A SARS-CoV protein, ORF-6, induces caspase-3 mediated, ER stress and JNK-dependent apoptosis. Biochim Biophys Acta, 2008. 1780(12): p. 1383-7.

90. BORAPPA MUTHUKALA, K.S., KAMALANATHAN ASHOK, IN SILICO DOCKING OF QUERCETIN COMPOUND AGAINST THE HELA CELL LINE PROTEINS. Int J Curr Pharm Res, 2014. 7(1): p. 13-16.

91. DeDiego, M.L., et al., Inhibition of NF-kappaB-mediated inflammation in severe acute respiratory syndrome coronavirus-infected mice increases survival. J Virol, 2014. 88(2): p. 913-24.

92. Oudkerk, M., et al., Diagnosis, Prevention, and Treatment of Thromboembolic Complications in COVID-19: Report of the National Institute for Public Health of the Netherlands. Radiology, 2020: p. 201629.

93. Chen, G., et al., Clinical and immunological features of severe and moderate coronavirus disease 2019. J Clin Invest, 2020. 130(5): p. 2620-2629.

94. Jose, R.J. and A. Manuel, COVID-19 cytokine storm: the interplay between inflammation and coagulation. Lancet Respir Med, 2020. 8(6): p. e46-e47.

95. Widodo, R.H.a.G.P., Molecular Docking Analysis: Interaction Studies of Natural Compounds to Antiinflammatory Targets. Quantitative Structure-activity Relationship, 2017.

96. Tang, J., et al., Quercetin and Quercitrin Attenuates the Inflammatory Response and Oxidative Stress in LPS-Induced RAW264.7 Cells: In Vitro Assessment and a Theoretical Model. Biomed Res Int, 2019. 2019: p. 7039802.

97. Fouache, A., et al., Flavonoids differentially modulate liver X receptors activity-Structure-function relationship analysis. J Steroid Biochem Mol Biol, 2019. 190: p. 173-182.

98. Medici, T.C. and P. Radielovic, Effects of drugs on mucus glycoproteins and water in bronchial secretion. J Int Med Res, 1979. 7(5): p. 434-42. 
99. Wang, J., et al., Synergism of ursolic acid derivative US597 with 2-deoxy-D-glucose to preferentially induce tumor cell death by dual-targeting of apoptosis and glycolysis. Sci Rep, 2014. 4: p. 5006.

100. Li, A.J., Q. Wang, and S. Ritter, Participation of hindbrain AMP-activated protein kinase in glucoprivic feeding. Diabetes, 2011. 60(2): p. 436-42.

101. Rutherford, C., et al., Phosphorylation of Janus kinase 1 (JAK1) by AMP-activated protein kinase (AMPK) links energy sensing to anti-inflammatory signaling. Sci Signal, 2016. 9(453): p. ra109.

102. Kuchipudi, S.V., The Complex Role of STAT3 in Viral Infections. J Immunol Res, 2015. 2015: p. 272359.

103. Mizutani, T., et al., Tyrosine dephosphorylation of STAT3 in SARS coronavirus-infected Vero E6 cells. FEBS Lett, 2004. 577(1-2): p. 187-92.

104. Li, X.X., et al., Determining the Balance Between Drug Efficacy and Safety by the Network and Biological System Profile of Its Therapeutic Target. Front Pharmacol, 2018. 9: p. 1245.

105. Chen, L., et al., A novel paradigm for potential drug-targets discovery: quantifying relationships of enzymes and cascade interactions of neighboring biological processes to identify drug-targets. Mol Biosyst, 2011. 7(4): p. 1033-41.

106. Muhammad, J., et al., Network Pharmacology: Exploring the Resources and Methodologies. Curr Top Med Chem, 2018. 18(12): p. 949-964.

107. Zhu F, H.L., Zheng C, What are next generation innovative therapeutic targets? Clues from genetic, structural, physicochemical, and systems profiles of successful targets. J Pharmacol Exp Ther, 2009. 330(1): p. 304-315.

108. Li YH, Y.C., Li XX, Therapeutic target database update 2018: enriched resource for facilitating benchto-clinic research of targeted therapeutics. Nucleic Acids Res, 2018. 46(D1): p. D1121-D1127.

109. Hart T, C.M., Aregger M, High-Resolution CRISPR Screens Reveal Fitness Genes and Genotype-Specific Cancer Liabilities. Cell, 2015. 163(6): p. 1515-1526.

\section{Competing Interests}

The authors declare no competing interests.

\section{Declarations}

Pankaj Khurana and Rajeev Varshney contributed equally and are equal first authors.

\section{Figures}




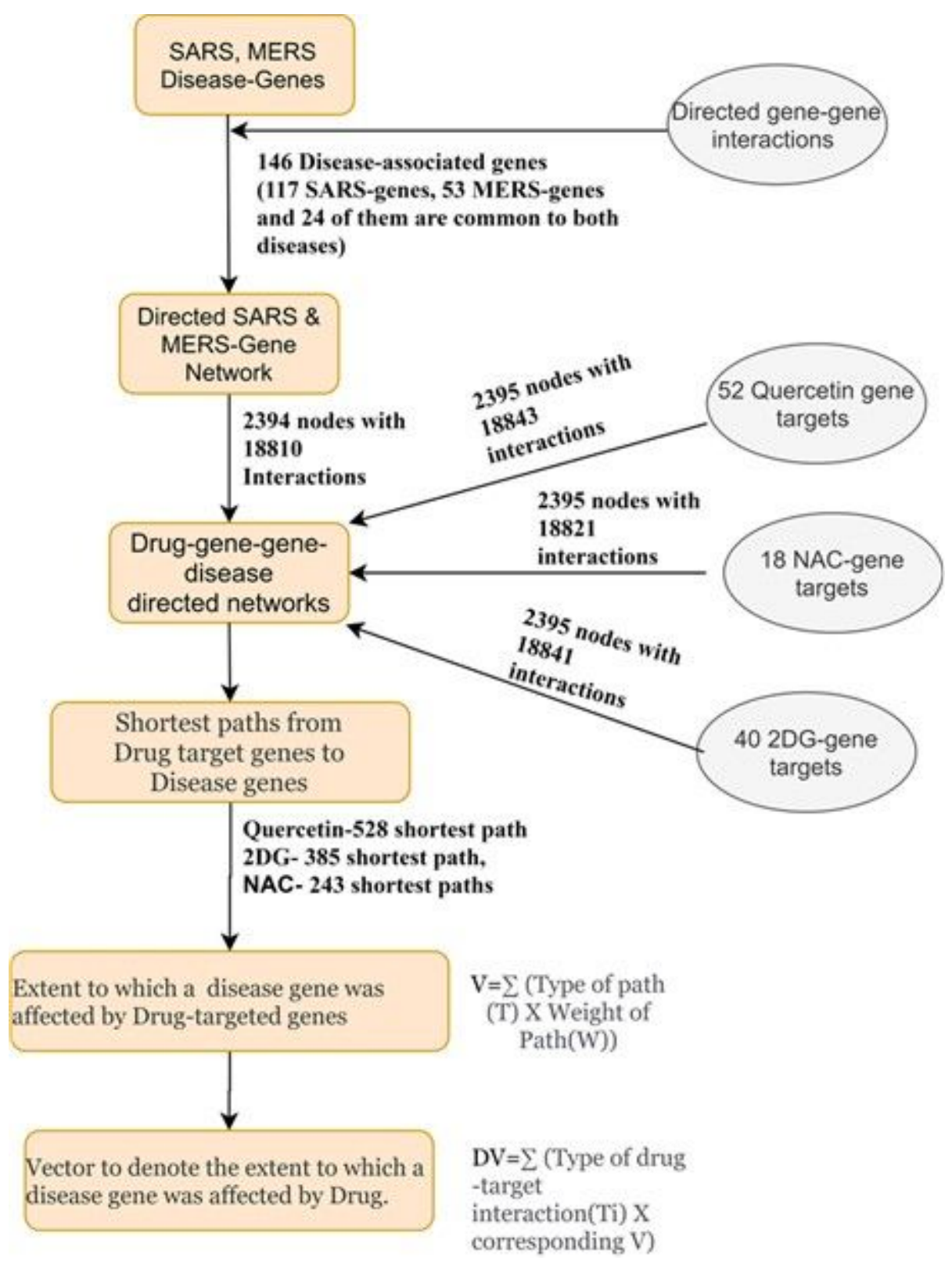

\section{Figure 1}

Graphical representation of methodology followed to compare Quercetin, NAC and 2-DG effectiveness against COVID-19 using DV vector

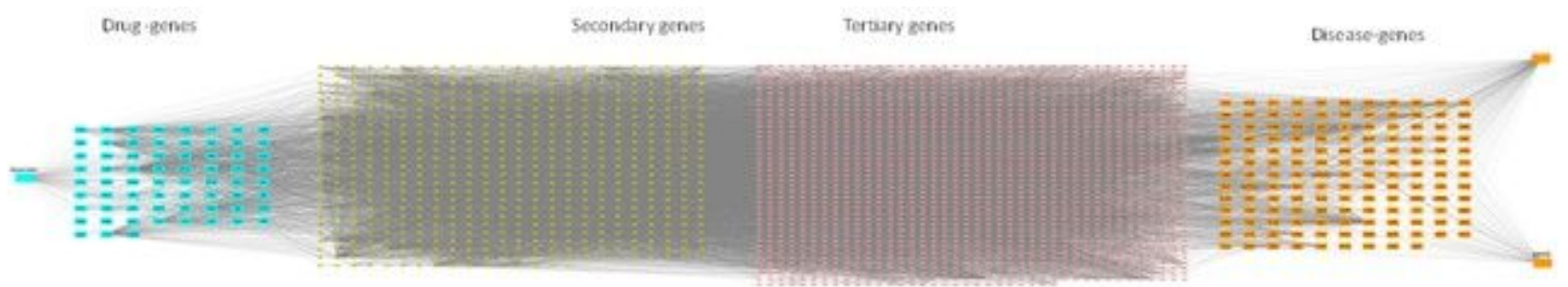

Figure 2

Quercetin-gene-gene-disease directed network. Quercetin direct gene interactors are highlighted as Cyan nodes. Its connecting secondary and tertiary genes are shown as green and pink nodes respectively. 
SARS and MERS with their direct gene interactors are shown as orange nodes.

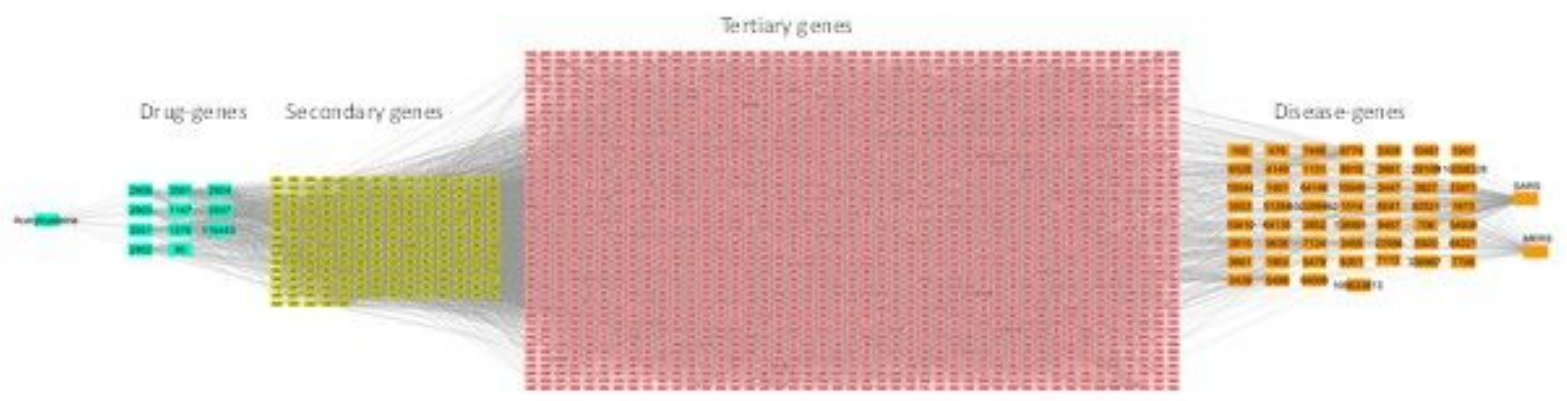

\section{Figure 3}

NAC-gene-gene-disease directed network. NAC direct gene interactors are highlighted as Cyan nodes. Its connecting secondary and tertiary genes are shown as green and pink nodes respectively. SARS and MERS with their direct gene interactors are shown as orange nodes.

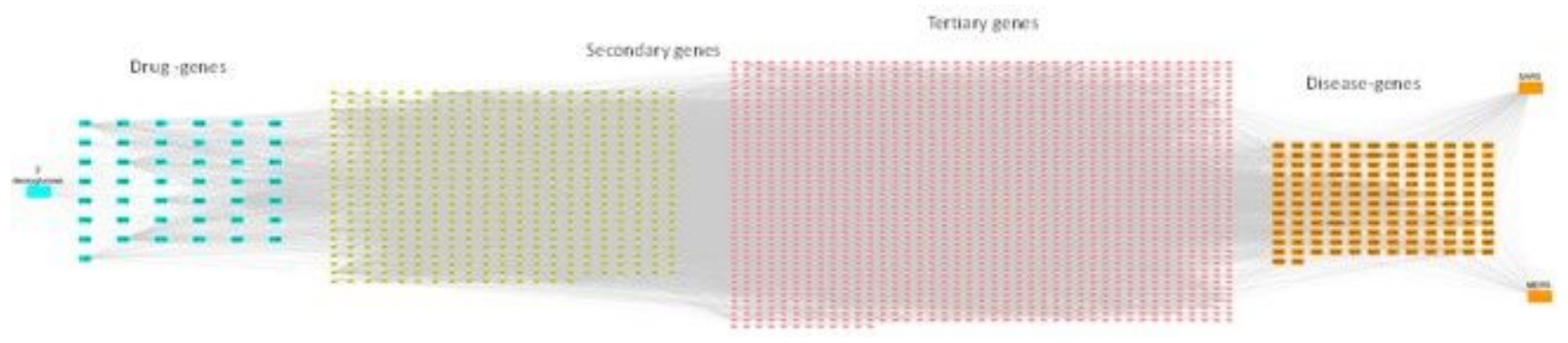

\section{Figure 4}

2-DG-gene-gene-disease directed network. 2-DG direct gene interactors are highlighted as Cyan nodes. Its connecting secondary and tertiary genes are shown as green and pink nodes respectively. SARS and MERS with their direct gene interactors are shown as orange nodes. 


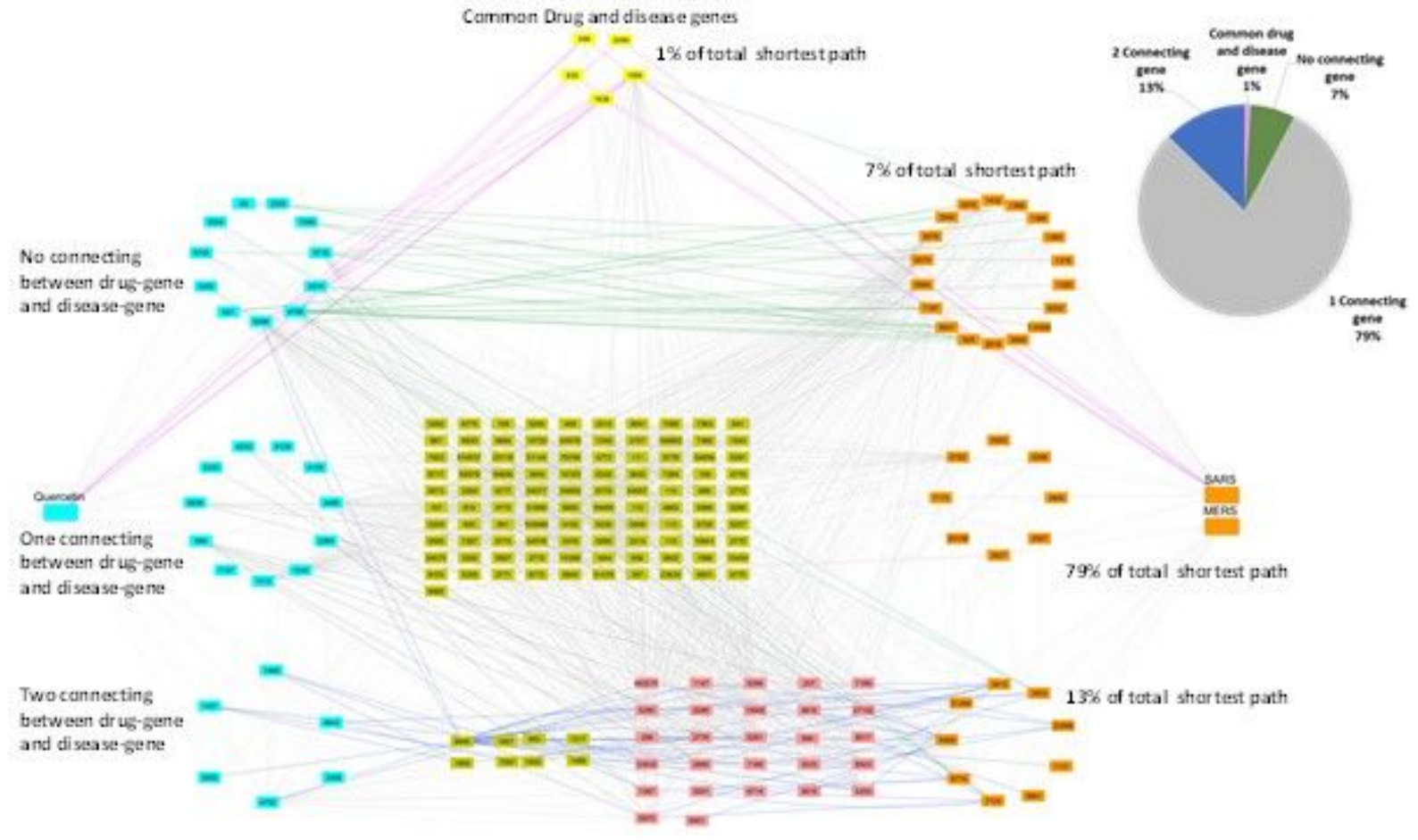

\section{Figure 5}

Quercetin-gene-gene-disease shortest path network. The drug and its direct interacting genes are highlighted as cyan nodes. The disease nodes and its direct interacting gene are highlighted as orange nodes. The genes that are common to both drug and disease have been shown as yellow nodes. The primary genes that lie on path having 1 interconnecting gene are shown as green nodes and secondary genes that lie on path having 2 interconnecting gens are shown as pink nodes. The pie-chart depicts the percentage of shortest path in each category of common, zero, one or two connecting genes in pink, green, grey and blue respectively. The respective color scheme is followed for the edge color in the network. 


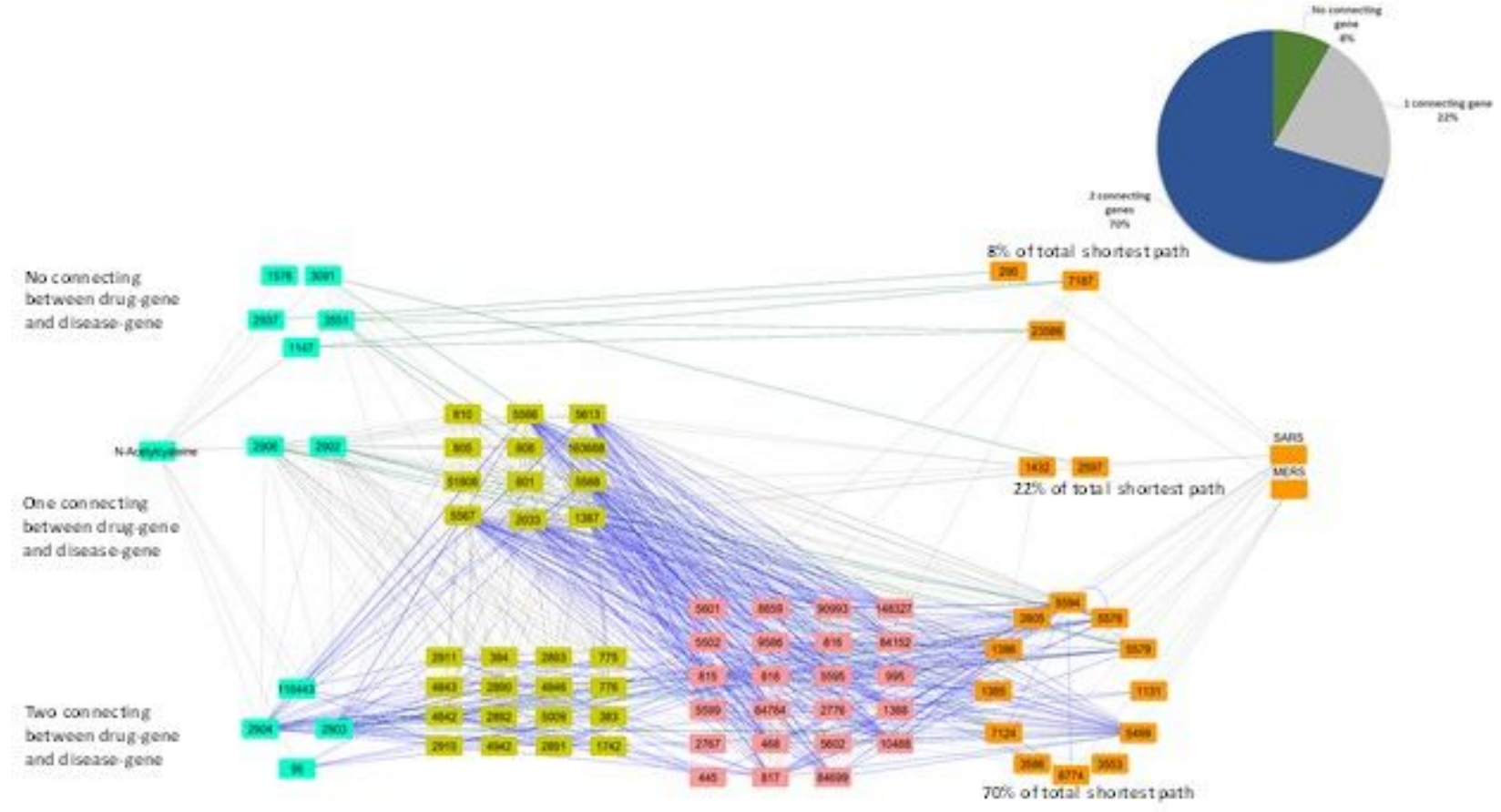

Figure 6

NAC-gene-gene-disease shortest path network. The drug and its direct interacting genes are highlighted as cyan nodes. The disease nodes and its direct interacting gene are highlighted as orange nodes. The primary genes that lie on path having 1 interconnecting gene are shown as green nodes and secondary genes that lie on path having 2 interconnecting gens are shown as pink nodes. The pie-chart depicts the percentage of shortest path in each category of zero, one or two connecting genes in green, grey and blue respectively. The respective color schema is followed for the edge color in the network.

No connect ing between druggene and disease-gene
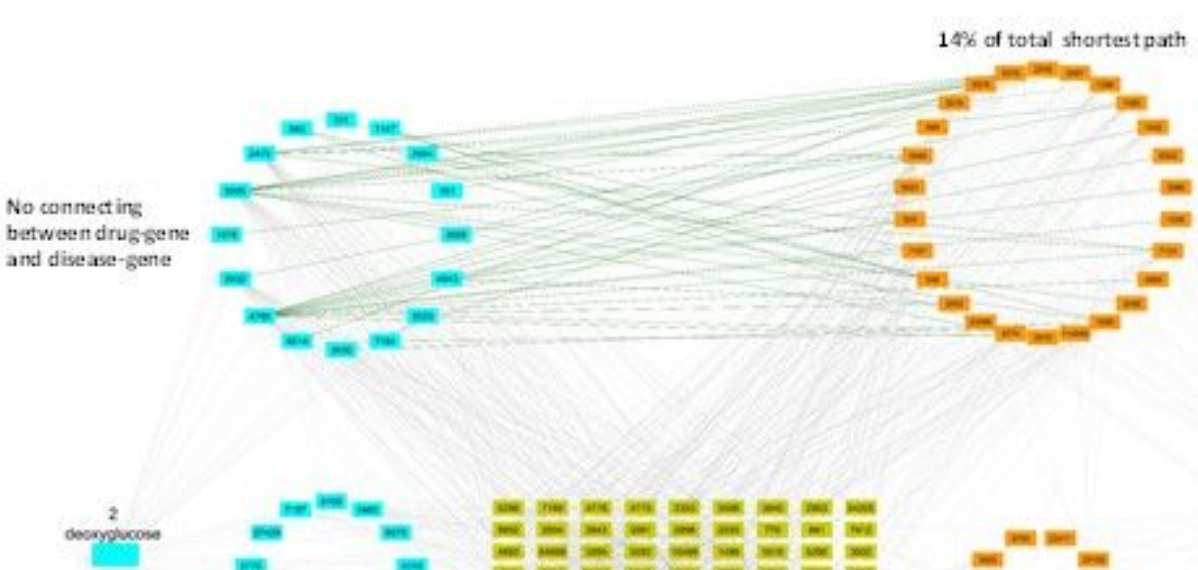

One cannexting between druggene and disease-gene

Twoconnecting between drusgene and disease-gene
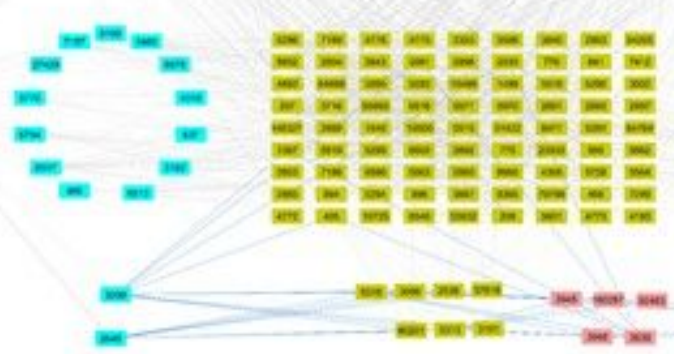

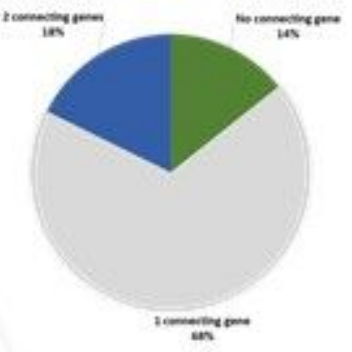

Figure 7 
2-DG-gene-gene-disease shortest path network. The drug and its direct interacting genes are highlighted as cyan nodes. The disease nodes and its direct interacting gene are highlighted as orange nodes. The primary genes that lie on path having 1 interconnecting gene are shown as green nodes and secondary genes that lie on path having 2 interconnecting gens are shown as pink nodes. The pie-chart depicts the percentage of shortest path in each category of zero, one or two connecting genes in green, grey and blue respectively. The respective color schema is followed for the edge color in the network.

\section{Parameters for Connectivity of the target}

Average Shortest Path

Length

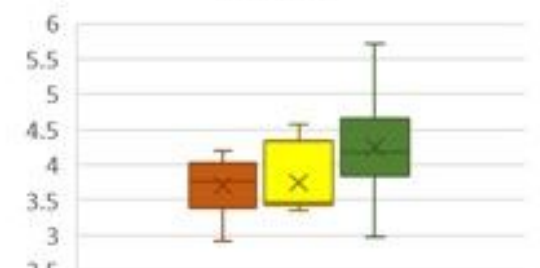

2.5
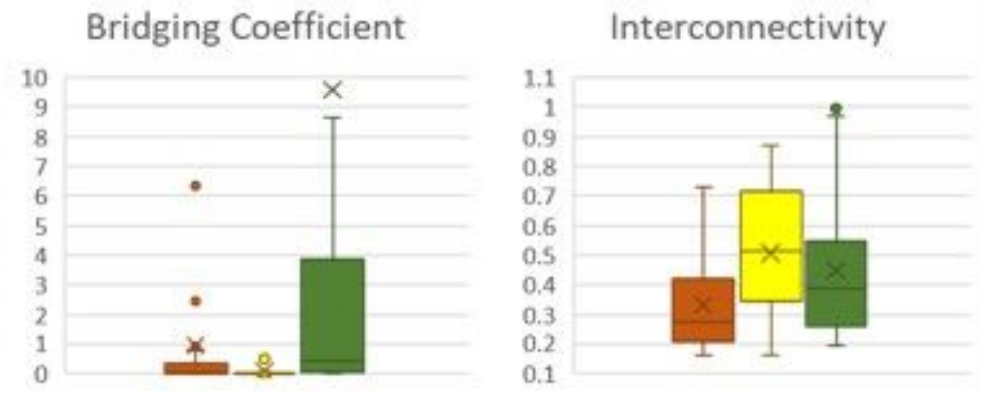

\section{Parameter for Centrality of the target}

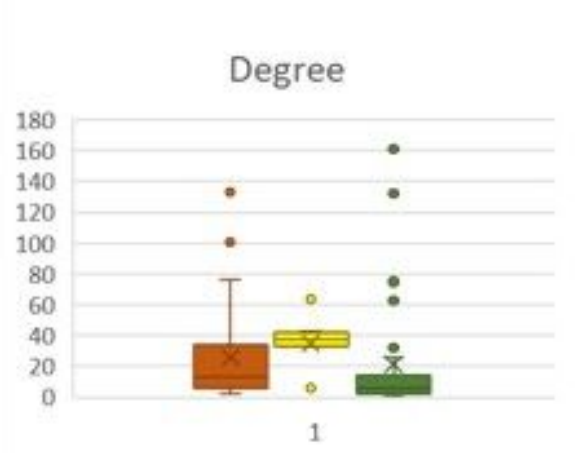

\section{Average Closeness}
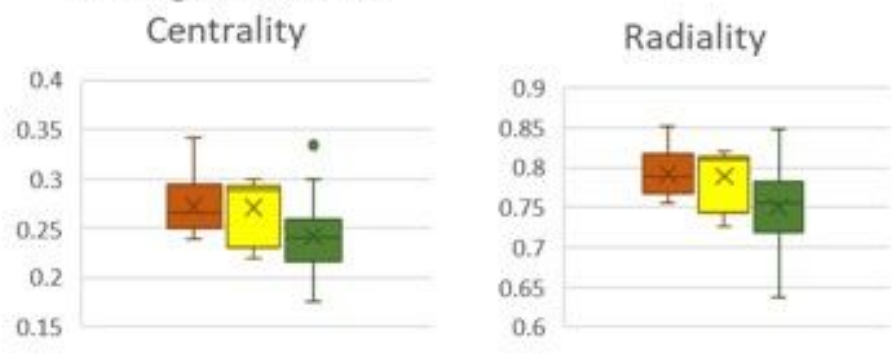

\section{Parameter for Human Biological System Features}

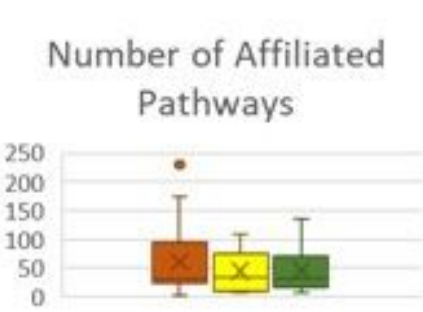

Number of Similarity

\section{Proteins}

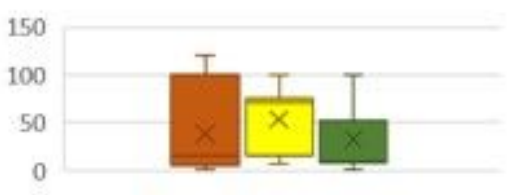

Percentage of direct interactors which are core-essential genes

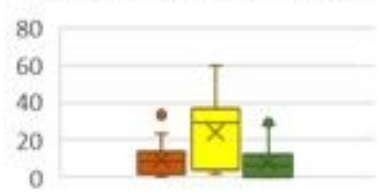

2-DG

NAC

Quercetin 
The parameters of connectivity of the target, centrality of the target and human biological features of the three drugs.

\section{Supplementary Files}

This is a list of supplementary files associated with this preprint. Click to download.

- supplementary1.xlsx

- supplementary2.xlsx

- supplemetary3.xlsx

- supplemetary4.xIsx

- supplemetary5.xlsx 